\title{
Sternal defect reconstruction using a double-barrel vascularized free fibula flap: a case report
}

\author{
Paula Rocha Gravina, Daniel K. Chang, James A. Mentz, Rami Paul Dibbs, Marco Maricevich \\ Division of Plastic Surgery, Michael E. DeBakey Department of Surgery, Baylor College of Medicine, Houston, TX, USA
}

Total and subtotal sternectomy oncological defects can result in large deficits in the chest wall, disrupting the biomechanics of respiration. Reviewing the current literature involving respiratory function and rib motion after sternectomy, autologous rigid reconstruction was determined to provide the optimal reconstructive option. We describe a novel technique for sternal defect reconstruction utilizing a double-barrel, longitudinally oriented, vascularized free fibula flap associated with rib titanium plates fixation. Our reconstructive approach was able to deliver a physiological reconstruction, providing rigid support and protection while allowing articulation with adjacent ribs and preservation of chest wall mechanics.

Keywords Sternal / Reconstruction / Fibula / Bone / Plastic
Correspondence: Marco Maricevich Division of Plastic Surgery, Michael E. DeBakey Department of Surgery, Baylor College of Medicine, 1977 Butler Blvd. Suite E6.100, Houston, TX 77030, USA

Tel: +1-713-798-6141

Fax: +1-713-798-2290

E-mail: marco.maricevich@bcm.edu

Received: April 26, $2021 \bullet$ Revised: June 3, $2021 \bullet$ Accepted: July 7, 2021

pISSN: 2234-6163 • elSSN: 2234-6171 • https://doi.org/10.5999/aps.2021.00682 • Arch Plast Surg 2021;48:498-502

\section{INTRODUCTION}

Total and subtotal sternectomy defects from oncologic resection are a challenging problem for the reconstructive surgeon. The three major goals of sternal reconstruction are to protect the underlying viscera, provide adequate tissue coverage that obliterates dead space, and restore stability of the anterior chest wall for effective pulmonary function [1].

Restoration of chest wall stability plays a crucial role in maintaining chest wall dynamics and allowing normal respiration. Nagasao et al. [2] utilized computer simulations to study the biomechanical changes in rib motion in a variety of simulated sternal defects. It was found that the functional loss in thoracic expansion is caused by a phenomenon termed the "hugging disorder," relating to the abnormal inward motion of the ribs without sternal support. This effect was most pronounced in total and subtotal sternal defects, significantly restricting the volume change of thoraces as low as $20 \%$ of normal values during respi- ration.

Current reconstructive algorithms for total and subtotal sternectomies favor a polymethylmethacrylate/polypropylene (PMM/PP) implant sandwich technique combined with an overlying soft tissue flap [3]. A titanium rib bridge system associated with soft tissue flaps has also been proposed in the literature with good results [4].

The vascularized fibula's strong cortical bone is excellent for holding screws, can integrate with local bone, and enjoys low risk of rejection $[5,6]$. With concern for long term delayed complications in a young active patient, possible infection associated with $\mathrm{PMM} / \mathrm{PP}$ and hardware failure with spanning rib plates alone, we propose a novel approach of rigid reconstruction for large sternectomy defects using a longitudinally oriented double-barreled free fibula flap in association with titanium plates and local soft tissue coverage. This technique provides a stable rigid reconstruction that effectively preserves chest wall biomechanics. 


\section{CASE}

A 31-year-old healthy and active male with a 3-month history of worsening midline chest pain was found to have a $4.5 \times 1.6 \mathrm{~cm}$ expansile lytic mass. Tissue biopsy revealed spindle-cell-neoplasm with myxoid features. Informed consent was obtained from the patient for surgical management. Ablative surgery resulted in subtotal resection of the sternum sparing only the manubrium. A free fibula flap was harvested in a double barrel fashion to best mimic the resected sternum. Minor trimming of the residual costochondral cartilages allowed a fit of both fibula bone segments side-by-side in the longitudinal axis. The fibula vascular pedicle was anastomosed to the internal mammary vessels at the right second rib space. Periosteal and cortical windows were created along the lateral edges of the inset fibula flap to allow direct contact with the costochondral cartilages of ribs 3,4 , and 5 bilaterally. Fixation of the ribs were performed using the Synthes-chest-wall-reconstruction $1.5 \mathrm{~mm}$ titanium plates across the fibula flap and remaining rib segments bilaterally along ribs 3, 4, and 5. Bicortical locking fixation was placed into bilateral osseous rib and monocortical locking fixation to both segments of the fibula at each level. Bilateral pectoralis flaps were then advanced and closed at the midline over two subcutaneous drains providing complete hardware coverage. Bilateral pleural chest tubes were placed (Fig. 1).

The patient was extubated at the end of the case and discharged home on postoperative day 11 with no supplemental oxygen, incentive spirometry levels above $2,000 \mathrm{~mL}$, adequate pain control, and no signs of chest wall instability or paradoxical motion.
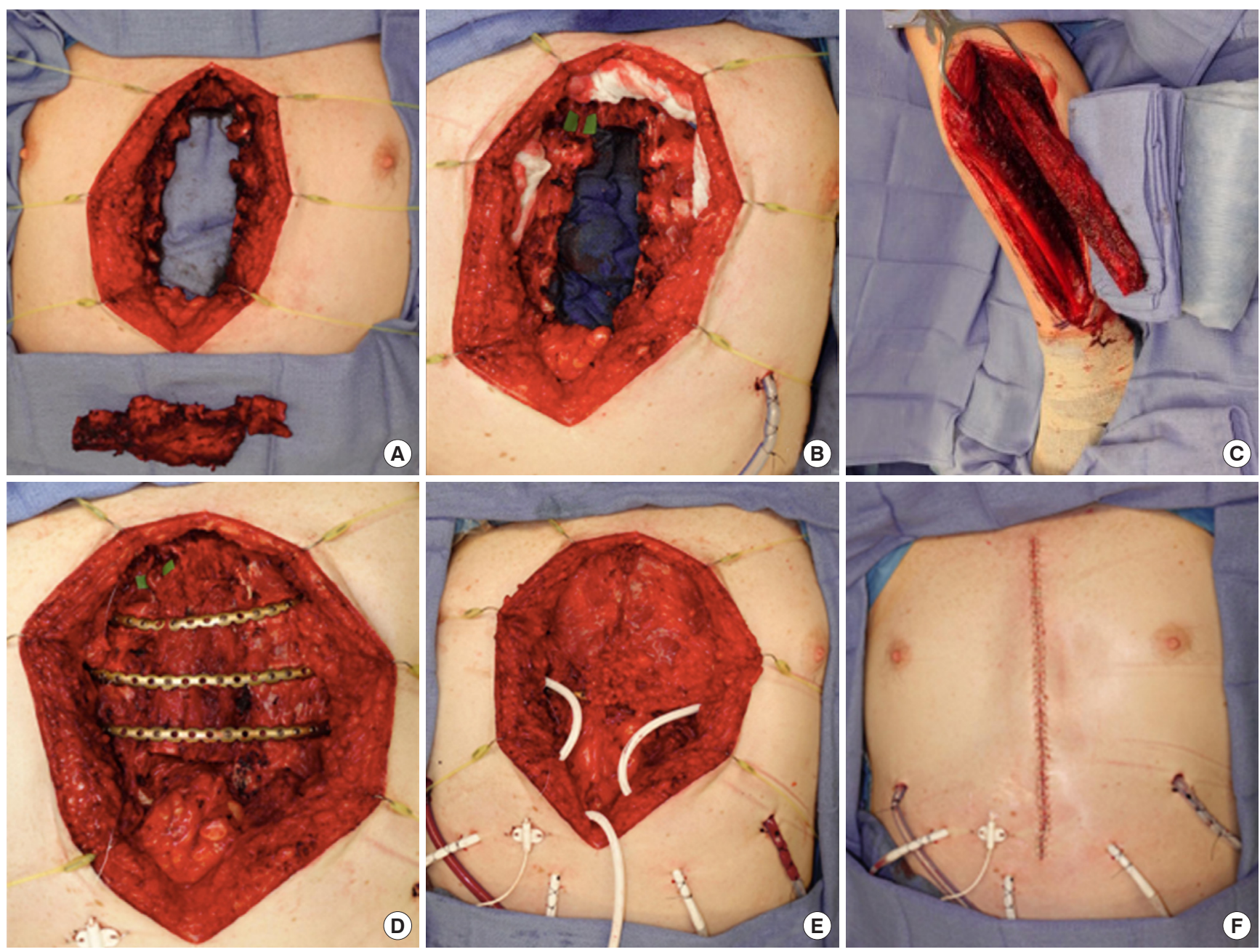

Fig. 1. Double-barrel vascularized free fibula flap harvest and transfer. (A) Sternal wound defect and specimen. (B) Right internal mammary artery and vein harvested from the second intercostal space. (C) Dissected left fibula free flap based on peroneal vessels. Preserving the vascular pedicle along its entire course, the bone was transected on its axial plane resulting in two segments of equal length; its distal segment was turned $180^{\circ}$ in a "double barrel" fashion before transfer to sternectomy defect. (D) Synthes titanium $1.5 \mathrm{~mm}$ chest-wall-reconstruction plates with screw fixation to bilateral ribs and to fibula bone flap. (E) Bilateral pectoralis major muscle flaps and epigastric adipofascial flap used to cover hardware. (F) Skin closure and internal Doppler for monitoring of the flap. 


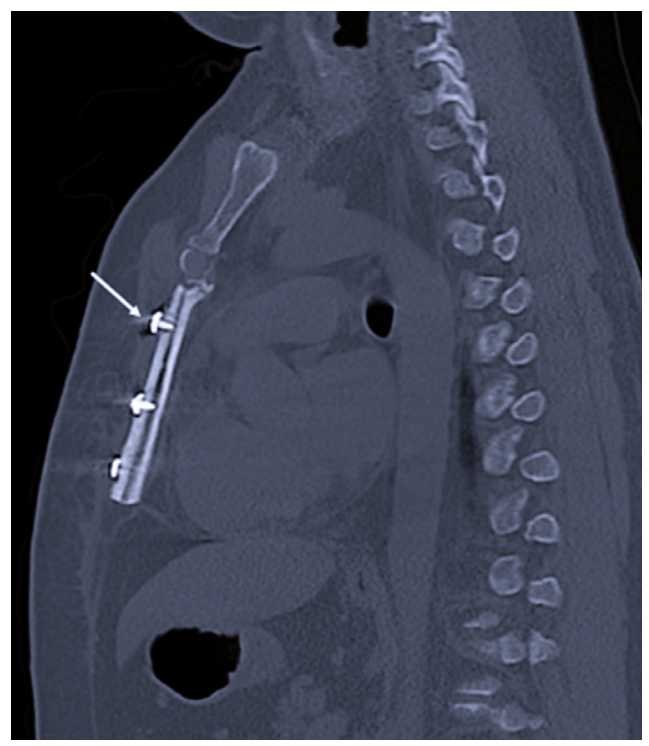

Fig. 2. Postoperative computed tomography (CT) scan demonstrating stable, rigid chest wall. CT scan (sagittal view) of chest 7 months postoperative, showing unicortical screws through each fibula segment at levels of ribs 3,4 , and 5 (white arrow).

Postoperative computed tomography scan of the chest at 5 and 7 months showed a stable rigid chest wall with well-maintained alignment of the fibula flap, titanium plates across 3,4 , and 5th ribs bilaterally (Figs. 2, 3). Both images showed no evidence of hardware failure. Clinically, he reports painless and effortless respiration. Excursions were symmetric bilaterally (Supplemental Video 1) with no evidence of chest wall instability or paradoxical motion.

\section{DISCUSSION}

Skeletal stabilization in total and subtotal sternectomy is a reconstructive challenge. While the current standard is prosthetic rigid stabilization, this is inevitably associated with a risk of infection and long-term failure $[7,8]$. The choice for this novel reconstruction technique was made jointly with this young active patient to address his specific anatomical deficits while achieving the aforementioned goals of sternal reconstruction.

Bone grafts are beneficial options for reconstruction because they are capable of incorporating into recipient tissue with revascularization and cellular repopulation, rendering them more resistant to postoperative infection [1]. While bony reconstruction has demonstrated positive outcomes, there is a paucity of data for its use in sternal defects [9]. In addition to vascularized fibula grafts, other autologous bone reconstruction options have been used to surgically manage large sternal defects for skeletal rigidity. In their case series, Haraguchi et al. [10] reported that three of their 15 patients underwent autologous bone grafting

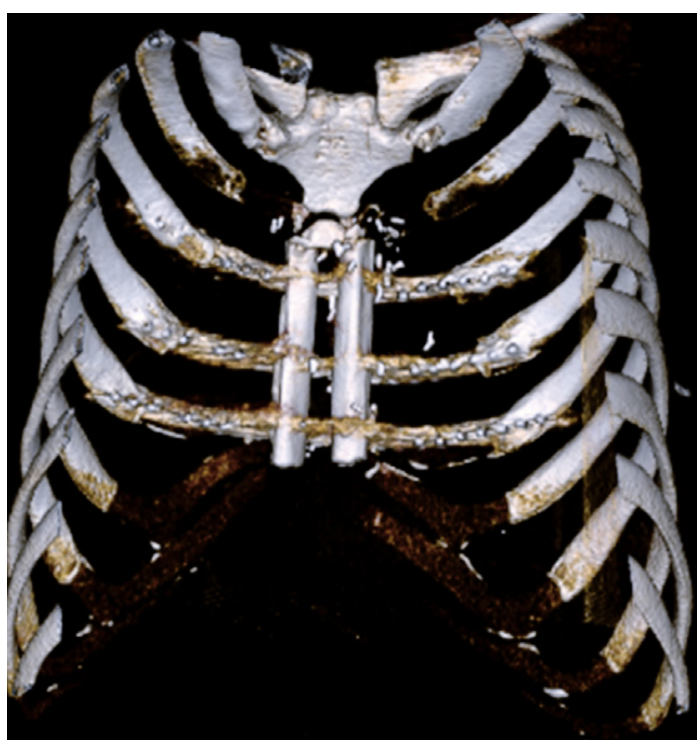

Fig. 3. Three-dimensional chest computed tomography (CT) scan demonstrating successful double barrel fibula free flap. Chest CT scan three-dimensional reconstruction showing a vertically oriented double barrel fibula free flap and titanium plates secured across both fibula segments and level of ribs 3, 4, and 5 .

(one for rib, one for rib and iliac crest, and one for iliac crest) following sternectomy for primary, recurrent breast carcinoma. While none of these patients demonstrated postoperative complications, none survived as a result of their cancer diagnosis. Drinnon et al. [9] reported a single case of a patient who successfully underwent sternal tumor resection and reconstruction with an iliac crest autograft with no major complications at 9 months follow-up. Beltrami and Gidaro [11] demonstrated good outcomes utilizing two split tibial grafts following resection of the manubrium, inner third of the left clavicle, and medial ends of the bilateral first two ribs. Nevertheless, while numerous anatomically distinct bones have been incorporated, larger prospective studies are needed to compare outcomes between different autologous bones for sternal reconstruction.

Considerations for vertical versus transverse orientation of the fibula were weighed, and the decision was made that a vertical orientation provides a more physiologic reconstruction of the sternum. While transverse orientation could theoretically allow for bone-to-bone healing, this would require additional resection of the ribs back to the osseous rib and limit the number of rib spaces that could be spanned [12]. Nahabedian et al. [13] performed allogenic fibula grafting following cardiac transplant and sternal resection to accommodate a larger heart that required greater thoracic volume for appropriate function. This technique involved dividing the fibula allograft into two $5 \mathrm{~cm}$ segments that were transversely oriented adjacent to ribs 8 and 10 with no major postoperative complications noted. Vertical 
orientation of the fibula placed between the rib spaces, however, provides a physical stop to prevent the "hugging effect" caused by the bilateral rib cages shifting inwards towards the midline.

Given that our fibula was transected and oriented $180^{\circ}$ in a "double barrel" fashion, there was concern for the vascular supply to the distal segment due to the anticipated folding of the pedicle. In our case, a $4 \mathrm{~cm}$ bone segment was removed from the osteotomy site to allow for a gentle curve of the vascular pedicle in between the proximal and distal segments of the fibula flap. The use of the contralateral internal mammary vessels as a super charge option is a possible alternative. An independent microvascular anastomosis recipient vessel for the distal segment of fibula could also be utilized, but intraoperative assessment of the distal segment of the fibula showed significant bleeding from its distal stump throughout the entire case.

The plates were chosen to help maintain position of the fibula, but as opposed to spanning plates used alone, the fibula as a physical stop helps to minimize the excessive rib motion that can lead to plate failure. Periosteal and cortical windows were made in the fibula at its junction with the adjacent cartilaginous ribs, to allow a degree of integration and fibrocartilaginous pseudo-joint formation that mimics the native costochondral joints. Experience in reconstruction throughout the body has shown us that autologous reconstruction can provide many benefits particularly in long-term outcomes. In the treatment of large bony defects, free fibula grafts have been shown to boast an impressive rate of union at $82 \%$ at 2 years and $97 \%$ at 5 years [14]. Due to this property, eventual hardware failure can be of no clinical significance if the bone graft has incorporated. In a series of 87 patients requiring removal of fixation plates after mandibular reconstruction using an osteocutaneous free flap, the flap was maintained in all but two patients [15]. In this way, our graft design will hopefully be able withstand the potential complications associated with plate use. We present this case report to increase consideration for autologous rigid reconstruction in oncologic sternal resections in the young patient population.

\section{NOTES}

\section{Conflict of interest}

No potential conflict of interest relevant to this article was reported.

\section{Ethical approval}

The study was performed in accordance with the principles of the Declaration of Helsinki. Written informed consent was obtained.

\section{Patient consent}

The patient provided written informed consent for the publication and the use of his images.

\section{Author contribution}

Conceptualization: M Maricevich. Data curation: PR Gravina, DK Chang. Methodology: PR Gravina. Project administration: M Maricevich. Visualization: M Maricevich. Writing - original draft: PR Gravina, DK Chang, JA Mentz, RP Dibbs. Writing review \& editing: PR Gravina, JA Mentz, RP Dibbs.

\section{ORCID}

Paula Rocha Gravina https://orcid.org/0000-0003-3865-4774

Daniel K. Chang https://orcid.org/0000-0001-8389-2996

James A. Mentz https://orcid.org/0000-0002-4840-8326

Rami Paul Dibbs $\quad$ https://orcid.org/0000-0002-2559-1711

Marco Maricevich https://orcid.org/0000-0003-3980-720X

\section{Supplementary material}

Supplemental Video 1. Postoperative physical exam demonstrating bilateral, symmetric breathing at 7 months postoperatively. Bilateral symmetric excursions with no paradoxical motion and excellent chest wall stability. Supplemental data can be found at: https://doi.org/10.5999/aps.2021.00682.v001.

\section{REFERENCES}

1. Sanna S, Brandolini J, Pardolesi A, et al. Materials and techniques in chest wall reconstruction: a review. J Vis Surg 2017;3:95.

2. Nagasao T, Kasai S, Shimizu Y, et al. A biomechanical study of relationship between sternum defect patterns and thoracic respiration. Comput Assist Surg (Abingdon) 2016;21:18.

3. Butterworth JA, Garvey PB, Baumann DP, et al. Optimizing reconstruction of oncologic sternectomy defects based on surgical outcomes. J Am Coll Surg 2013;217:306-16.

4. Fabre D, El Batti S, Singhal S, et al. A paradigm shift for sternal reconstruction using a novel titanium rib bridge system following oncological resections. Eur J Cardiothorac Surg 2012;42:965-70.

5. Arai K, Toh S, Tsubo K, et al. Complications of vascularized fibula graft for reconstruction of long bones. Plast Reconstr Surg 2002;109:2301-6.

6. Minami A, Kasashima T, Iwasaki N, et al. Vascularised fibular grafts: an experience of 102 patients. J Bone Joint Surg Br 2000;82:1022-5.

7. Grabert S, Erlebach M, Will A, et al. Unexpected results af- 
ter sternal reconstruction with plates, cables and cannulated screws. Interact Cardiovasc Thorac Surg 2016;22:663-7.

8. Cicilioni OJ Jr, Stieg FH 3rd, Papanicolaou G. Sternal wound reconstruction with transverse plate fixation. Plast Reconstr Surg 2005;115:1297-303.

9. Drinnon KD, Sherali S, Cox CT, et al. Sternal tumor resection and reconstruction using iliac crest autograft. Plast Reconstr Surg Glob Open 2020;8:e3002.

10. Haraguchi S, Hioki M, Hisayoshi T, et al. Resection of sternal tumors and reconstruction of the thorax: a review of 15 patients. Surg Today 2006;36:225-9.

11. Beltrami V, Gidaro G. Resection and reconstruction of the sternum: case report. Thorax 1976;31:350-3.
12. Ozkan S. The reconstruction of the anterior thorax wall after sternal resection through the combination of autologous fibula graft and metal plate. EJMO 2018;2:243-6.

13. Nahabedian MY, Riley LH, Greene PS, et al. Sternal stabilization using allograft fibula following cardiac transplantation. Plast Reconstr Surg 2001;108:1284-8.

14. Houdek MT, Bayne CO, Bishop AT, et al. The outcome and complications of vascularised fibular grafts. Bone Joint J 2017;99-B:134-8.

15. Wood CB, Shinn JR, Amin SN, et al. Risk of plate removal in free flap reconstruction of the mandible. Oral Oncol 2018;83:91-5. 